\title{
Discriminating Borderline Ovarian Tumors from Ovarian Cancer: Focus on Systemic Inflammatory Response Markers
}

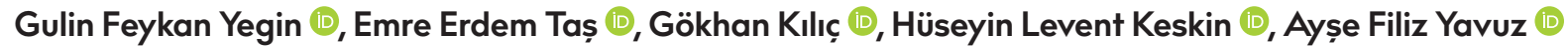 \\ Department of Gynecology and Obstetrics, Yıldırım Beyazıt University Ataturk Training and Research Hospital, Ankara, Turkey
}

ORCID IDs of the authors: G.F.Y. 0000-000I-8006-5055; E.E.T. 0000-000I-6043-2700; GK 0000-0002-9940-7139; H.L.K. 0000-0002-2268382l; A.F.Y. 0000-0003-3699-7757.

Cite this article as: Yeğin GF, Taș EE, Kılıç G, Keskin HL, Yavuz AF. Discriminating Borderline Ovarian Tumors from Ovarian Cancer: Focus on Systemic İnflammatory Response Markers. Cyprus J Med Sci 2020; 5(I): 36-40.

\section{BACKGROUND/AIMS}

This study aims to investigate the preoperative diagnostic accuracy of systemic inflammatory response (SIR) markers, including neutrophil-to-lymphocyte ratio (NLR) and platelet-to-lymphocyte ratio (PLR), in discriminating borderline ovarian tumors (BOTs) from malignancy and, thereby, prevent over-or underdiagnosis in the management of BOTs and ovarian malignancy.

\section{MATERIAL and METHODS}

Medical records of 99 patients who underwent surgical treatment and had confirmed histopathologic diagnosis of primary malignant or BOT were retrospectively analyzed. The recommended cut-off values for preoperative NLR and PLR were determined using receiver operating characteristic. The associations of NLR and PLR with tumors' malignancy potentials were analyzed using the Chi-square test or the Fisher's exact test.

\section{RESULTS}

The mean NLR and PLR were significantly lower ( $p=0.002$ and $p=0.006$, respectively) in BOTs group than in the epithelial ovarian carcinoma (EOC) group. Optimal cut-off points of NLR and PLR for discriminating BOTs and EOC group was 2.42 and 169.2 , respectively. The likelihood of malignancy increased in group with NLR values $>2.42$ ( $p<0.001 ; O R, 2.36,95 \% ; C l, 1.19-4.68$ ) and PLR values $>169.2$ ( $p<0.001$; $\mathrm{OR}, 3.6,95 \% ; \mathrm{Cl}, \mathrm{I.48}-8.76$ ). Most importantly, both NLR and PLR values were above the cut-off point, and the malignancy risk had a I2-fold increase ( $\mathrm{p}<0.00 \mathrm{I} ; \mathrm{OR}, 12.15,95 \% ; \mathrm{Cl}, 1.78-82.6)$.

\section{CONCLUSION}

This data will strengthen the discrimination of malignant tumors from BOTs and facilitate the decision-making on surgical radicality and may also be used in combination with imaging strategies, tumor markers, and frozen section to increase diagnostic accuracy.

Keywords: Borderline ovarian tumor, neutrophil-to-lymphocyte ratio, platelet-to-lymphocyte ratio, systemic inflammatory response

\section{INTRODUCTION}

Epithelial ovarian cancer is the most lethal gynecologic cancer; it is a major cause of cancer-related death in women (I, 2). The high mortality is associated with difficulties regarding early diagnosis, development of resistance to chemotherapeutic agents in advanced stage, and high recurrence rates (I). Currently, about $60 \%-65 \%$ of patients are being diagnosed with stage III ovarian carcinoma, which explains the high mortality of this neoplasm $(3,4)$. The five-year survival for patients diagnosed with stage I ranges from 80\% to 90\%; whereas for patients with stages III-IV, it ranges from 5\% to 50\% (4). Though great efforts had been made in decades, the abilities of predicting malignancy potential of ovarian tumors are inadequate, and there is still no well-established screening tool for ovarian cancer $(5,6)$.

Borderline tumors of the ovary (BOTs), also called tumors of low-malignant potential, are a heterogeneous group of lesions histologically defined by atypical epithelial proliferation without stromal invasion (7). The behavior of these noninvasive neoplasms is distinct from low-grade ovarian carcinoma, and they are considered a distinct clinical entity (7). BOTs account for $14 \%-15 \%$ of all primary ovarian neoplasms (8). The disease has a good prognosis (stage I five-year survival is $99 \%$ ), and unilateral salpingo-oophorectomy (USO) appears to be an option for women with unilateral disease (9). Com- 
plete staging with total abdominal hysterectomy and bilateral salpingo-oophorectomy ( $\mathrm{TAH}-\mathrm{BSO}$ ) is required for women with stage II or higher disease. The efficacy of conservative surgery in early-stage diseases was best illustrated in a systematic review and meta-analysis of 120 mostly retrospective studies (I0). For women with stage I ovarian borderline tumors treated with either USO or ovarian cystectomy, with an average follow-up of three to six years, the borderline recurrence rate was $13 \%$, recurrence with malignant disease was $1.6 \%$, and the death rate was $0.5 \%(10,11)$. According to recent data, for women with an apparent unilateral stage I BOT, unilateral salpingo-oophorectomy, pelvic washing, or omental and peritoneal biopsy is suggested rather than complete staging surgery $(I, 5)$. The ability to preoperatively distinguish borderline tumors from early-stage carcinoma considerably influences surgical treatment and allows improved counseling of patients.

Systemic inflammatory response (SIR) are closely associated with cancer initiation, progression, and metastasis, and thus, inflammatory markers, including the neutrophil-to-lymphocyte ratio (NLR) and platelet-to-lymphocyte ratio (PLR), have been studied and found to be related to cancer mortality and employed as useful prognostic indications in many solid tumors ( 12 , 13). Studies associated with BOTs are exactly limited, and to the best of our knowledge, the cut-off values of NLR and PLR to discriminate BOTs from malignant tumors have not been clarified yet. Thus, we conducted the current study to compare preoperative diagnostic accuracy of SIR markers, including NLR and $P L R$, in BOTs and malignant tumors, and thereby prevent overor underdiagnosis in the management of BOTs and ovarian malignancy.

\section{MATERIAL and METHODS}

\section{Subjects}

A retrospective analysis was performed with data gathered from 99 patients who underwent surgical treatment and were histopathologically diagnosed with primary malignant or BOT between 2007 and 2017. The indication criteria for surgical treatment (laparotomy/laparoscopy) was determined as anechoic cysts with a maximum diameter more than $7 \mathrm{~cm}$; persistence of change more than six months; altered CA 125, CA I5-3, CEA, alpha-fetoprotein, and CA 19.9; presence of intracystic vegetation; ovarian masses with septation and/or solid component; and resistance index by color Doppler less than or equal to 0.4 . On the

\section{Main Points:}

- The mean neutrophil-to-lymphocyte ratio (NLR) and platelet-to-lymphocyte ratio (PLR) were significantly lower $(p=0.002$ and $p=0.006$, respectively) in borderline ovarian tumors (BOTs) groups than in the epithelial ovarian cancer (EOC) group.

- Most importantly, the malignancy risk had a 12-fold increase in cases whom NLR and PLR values were both above the cut-off point $(p<0.00 I$; OR, 12.15, 95\%; Cl, I.7882.6).

- NLR and PLR can be used to strengthen the diagnostic accuracy on discriminating borderline ovarian tumors (BOTs) from ovarian cancer. other hand, the exclusion criteria suggests the presence of acute inflammatory disease, myeloproliferative disorders, concomitant gynecological and other cancers, autoimmune disease, and usage of any drug that affects CBC parameters, including anticoagulants or hormonal contents, or reported smoking. According to inclusion and exclusion criteria, 99 patients were enrolled in the study. All participants submitted a written informed consent. The study was approved by the Ethical Review Board of our institution. This research was conducted in accordance with the World Medical Association Declaration of Helsinki (and the 2000 revision).

Demographic and pathologic characteristics (age, gravidity, parity, histological type, FIGO stage) and data regarding preoperative SIR markers, including NLR and PLR, were retrieved from patients' medical files and hospital records. Blood samples were collected from patients during their admission at the hospital for surgery and before receiving any medications (Sysmex XE-2100 Automated CBC Analyzer, Sysmex, Nürnberg, Germany).

After grouping patients according to malignancy potential (i.e., borderline vs malignant epithelial ovarian tumors), the groups were compared in terms of the examined parameters.

\section{Data Extraction}

Preoperative blood samples were drawn 7-10 days prior to surgery. NLR was defined as absolute neutrophil count divided by absolute lymphocyte count, and PLR was defined as absolute platelet count divided by absolute lymphocyte count. Patients were then classified into low- and high-risk groups according to the cut-off values determined by the Youden index to examine the correlation between those markers and malignancy potential of epithelial ovarian tumors, preoperatively.

\section{Statistical Analyses}

The Kolmogorov-Smirnov assesses the normality of the data. Normally distributed data were expressed as means and standard deviations. Nonparametric data were expressed as medians and interquartile ranges. The groups were compared using independent sample t-tests and Mann-Whitney $U$ tests. Variables with a $p<0.05$ were included in the receiver operating characteristic (ROC) curve analysis which determines the cutoff values. The biggest Youden index (sensitivity+specificity-l) was selected as the optimal cut-off point. The associations of NLR and PLR with tumor malignancy potentials were analyzed using the Chi-square test or the Fisher's exact test.

Statistical analyses were conducted using the Statistical Package for the Social Sciences software for Windows version 21.0 (IBM Corporation, Armonk, NY, USA). Odds ratios and 95.0\% confidence intervals were determined. A $p<0.05$ was considered statistically significant.

\section{RESULTS}

The cases involved in the present study include a total of 99 patients; 79 (79.8) patients manifested epithelial ovarian carcinoma (EOC) and 20 (20.2\%) patients with borderline ovarian tumor (BOTs). In the BOTs group, 10 (I0.1\%) of the neoplasms were serous and 10 (10.1\%) were musinous. Demographic and pathologic characteristics and examined parameters of patients were summarized in Table I. 
TABLE I. Demographic and surgical characteristics and preoperative SIR markers including NLR and PLR of patients

\begin{tabular}{|c|c|c|c|}
\hline Characteristic & BOTs & EOC & $p$ \\
\hline \multicolumn{4}{|c|}{ Demographic and surgical, mean \pm SD (range) } \\
\hline Age (years) & $47.4 \pm \mid 4.5$ & $57.1 \pm \mid 4.5$ & 0.740 \\
\hline Gravidity & $4.24 \pm 2.86$ & $2.75 \pm 1.9$ & $0.042^{*}$ \\
\hline Parity & $2.00 \pm 1.30$ & $3.29 \pm 2.20$ & $0.002^{*}$ \\
\hline \multicolumn{4}{|l|}{ FIGO stage, $n(\%)$} \\
\hline Low stage (IA-IB) & $14(14.1 \%)$ & $3(3 \%)$ & $<0.00 l^{*}$ \\
\hline High stage (IC- II-III-IV) & $6(6.1 \%)$ & $76(76.8 \%)$ & $<0.00$ I $^{*}$ \\
\hline \multicolumn{4}{|c|}{ SIR markers, mean $\pm S D$ (range) } \\
\hline $\operatorname{NLR}(\%)$ & $2.23 \pm 0.83$ & $3.89 \pm 2.16$ & $0.002^{*}$ \\
\hline $\operatorname{PLR}(\%)$ & $151.714 \pm 75.210$ & $249.153 \pm 139.010$ & $0.006^{*}$ \\
\hline \multicolumn{4}{|c|}{$\begin{array}{l}\text { NLR, neutrophil-to-lymphocyte ratio; PLR, platelet-to-lymphocyte ratio; } \\
\text { BOT, borderline ovarian tumors; EOC, epithelial ovarian cancer; OR: odd } \\
\text { ratio; Cl: confidence interval }\end{array}$} \\
\hline
\end{tabular}

TABLE 2. Demographic and surgical characteristics and preoperative SIR markers including NLR and PLR of patients

\begin{tabular}{|lcccccc} 
& & $<$ cut-off & $>$ cut-off & P & OR & $95 \% \mathrm{Cl}$ \\
\hline NLR & EOC & $23(29.1 \%)$ & $56(70.9 \%)$ & $<0.001$ & 2.36 & $1.19-4.68$ \\
& BOT & $14(70 \%)$ & $6(30 \%)$ & & & \\
PLR & EOC & $22(27.8 \%)$ & $57(72.2 \%)$ & $<0.001$ & 3.6 & $1.48-8.76$ \\
& BOT & $16(80 \%)$ & $4(20 \%)$ & & & \\
NLR \& PLR & EOC & $31(39.2 \%)$ & $48(60.8 \%)$ & $<0.001$ & 12.15 & $1.78-82.6$ \\
& BOT & $19(95 \%)$ & $1(5 \%)$ & & & \\
& & &
\end{tabular}

NLR: neutrophil-to-lymphocyte ratio; PLR: platelet-to-lymphocyte ratio; BOT: borderline ovarian tumors; EOC: epithelial ovarian cancer; OR, odd ratio; $\mathrm{Cl}$, confidence interval

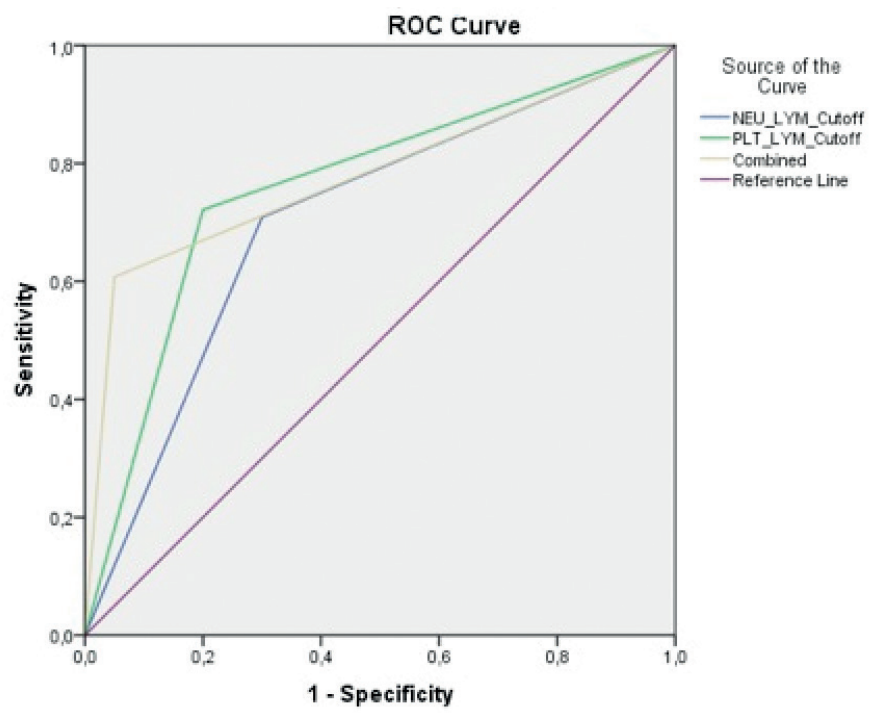

FIGURE I. The receiver operating characteristic curve analysis of the relationship between only NLR, only PLR, and both NLR and PLR values

NEU_LYM : neutrophil-to-lymphocyte ratio

PLT_LYM: platelet-to-lymphocyte ratio

Combined: both neutrophil-to-lymphocyte ratio and platelet-to-lymphocyte ratio neutrophil-to-lymphocyte ratio (NLR) and platelet-to-lymphocyte ratio (PLR) in discriminating borderline ovarian tumors (BOTs)
There was no significant difference in the demographic characteristics (age, gravidity, and parity) between the BOTs and EOC groups (all $p>0.05$ ).

The mean NLR and PLR were significantly lower ( $p=0.002$ and $p=0.006$, respectively) in BOTs group than in the EOC group.

ROC curve analyses revealed that the optimal cut-off points of NLR and PLR for discriminating BOTs and EOC group was 2.42 and 169.2, respectively (Figure I).

Finally, when the groups (i.e., BOTs and EOC) were subdivided according to NLR, PLR, and NLR-PLR cut-off values (NLR $\leq 2.42$ vs. NLR>2.42 and $P L R \leq 169.2$ vs. PLR>169.2), the ratio of patients whose NLR, PLR, and NLR-PLR were above the cut-off value were high in EOC group than BOTs group, and significant differences were observed $(p<0.001)$ (Table 2 ).

\section{DISCUSSION}

Our study demonstrated that in the group of patients with NLR values $>2.42$, the likelihood of malignancy increased by 2.3 -fold $(p<0.001 ; O R, 2.36,95 \% ; C l, 1.19-4.68)$, and the likelihood of malignancy of the ovarian tumor increased by 3.6 -fold in the patient group with PLR values >169.2 ( $p<0.001$; OR, 3.6, 95\%; Cl, I.48-8.76). Most importantly, both NLR and PLR values were above the cutoff point, and a 12 -fold increase in the malignancy risk was observed ( $p<0.001 ; O R, 12.15,95 \% ; C l, 1.78-82.6)$.

The increasing evidences focus on the importance of inflammation in the initiation, promotion, invasion, and metastasis periods of cancer. Increased neutrophil, platelet, C-reactive protein, and fibrinogen concentration in cancer process have been discussed in many studies (I4). In addition, neutrophil-to-platelet ratio (NLR) and platelet-to-lymphocyte ratio have also been suggested to be useful in discriminating malignant and benign ovarian tumors (15-19). Numerous studies have demonstrated the association of PLR and NLR with ovarian cancers (20-22). In this study, the cut-off values of these markers discriminate BOTs from malignancy, preoperatively, to prevent higher morbidity and mortality due to radical or second-look surgeries.

In a published meta-analysis, NLR and PLR were found to be significant predictors for solid tumors originating from several tissues (23). However, there is no consensus on predictive values of NLR and PLR in gynecologic cancers. Additionally, data pertaining to BOTs is limited (24).

Current tools used in the management of adnexal masses are gynecologic examination, tumor markers, and imaging methods. However, there are no sonographic features strongly suggestive of borderline histology, and sonographic appearance ranges from unilocular cysts to masses with solid components. Tumor markers have been demonstrated as useful in identifying malignancy, but these parameters are also found to be elevated in $25 \%-60 \%$ of BOTs (25-28). In current literature, CA 125, CEA, and CA 19-9 were targeted to identify the prognosis of borderline tumors but are not considered to be a diagnostic tool (29). Consequently, we are still unable to anticipate malignancy potential and invasion status of ovarian tumors, preoperatively. 
Intraoperative frozen section plays the most critical role in the management of these cases. However, the sensitivities for detection of BOTs are $50 \%-85 \%$. A previous study was designed with patients who underwent surgery due to an adnexal mass. Among these patients, $17.5 \%$ were preliminarily diagnosed as a BOT. The final pathology was reported as benign. More importantly, in $17.5 \%$ of patients diagnosed with ovarian carcinoma in frozen section, the final pathology was reported as BOT (24). All under- and overdiagnosis of these patients will undoubtedly cause mismanagement and increased morbidity and mortality due to overtreatment or recurrent surgeries.

Parameters that increase the frozen section's accuracy have also been studied, and the most favorable marker in literature was determined to be CA 125, especially in serous malignancies (25-27). Conversely, numerous studies showed no remarkable effect of CA 125 in increasing frozen section diagnostic potential (30). It has been reported that simple indices, such as NLR and $P L R$, have high sensitivity for detection of early-stage invasive ovarian cancer (3I). Additionally, the NLR value was found to be useful in increasing frozen section accuracy (24).

Although studies focus on the discrimination of malignant tumors from benign masses, it is also important to classify a tumor as borderline/malignant tumor especially in a fertility-preserving surgery and manage operation in an optimal way by decreasing morbidity and mortality.

In a reported study, a statically significant impact for both preoperative NLR and PLR in distinguishing BOTs from simple ovarian serous cysts was suggested (17). In another study designed on a patient group with benign and malignant ovarian tumors, preoperative PLR and platelet count were statistically found to be significant between groups, but there was no association detected with NLR (20). Similarly, in another study, preoperative NLR, PLR, and monocyte were established to be higher in malignant cases. It was also stated by authors that NLR and PLR combined with CA 125 can be useful for the differentiation of ovarian tumors, whether it's benign or malignant (32). PLR was also found to be correlated with low survival rates in ovarian cancers (19). In a study regarding discrimination of BOTs from malign tumors by using NLR and PLR, authors stated that these SIR markers are predictors of malignant tumors but not borderline tumors, even in case of tumor invasion less than $5 \mathrm{~mm}$ (33).

The current research is limited to its retrospective and single-centered design; thus, prospective randomized studies with larger sample sizes are required to verify our results.

In conclusion, this data will strengthen the elimination of malignant tumors from BOTs and may also be used in combination with imaging strategies, tumor markers, and frozen section to increase diagnostic accuracy before radical surgical interventions.

Ethics Committee Approval: Ethics committee approval was received for this study from the ethics committee of Yıldırım Beyazit University Faculty of Medicine Clinical Research Ethics Committee. (Approval date: 21.12.2018, approval no: $2367996 /$ i32).

Informed Consent: Written informed consent was obtained from patients who participated in this study.
Peer-review: Externally peer-reviewed.

Author contributions: Concept-N.B., B.S.; Design- B.S.,O.Y; SupervisionN.B.; Resource:- B.S.,O.Y; Materials- N.B.; Data Collection and/or Processing- B.S.,O.Y.; Analysis and/or Interpretation- B.S.,O.Y; Literature Search- B.S.,O.Y; Writing- B.S.,O.Y.; Critical Reviews- N.B.

Conflict of Interest: The authors have no conflicts of interest to declare.

Financial Disclosure: The authors declared that this study has received no financial support.

\section{REFERENCES}

I. Kwon BS, Jeong DH, Byun JM, Lee TH, Choi KU, Song YJ, et al. Prognostic value of preoperative lymphocyte-monocyte ratio in patients with ovarian clear cell carcinoma. J Cancer 2018; 9(7): I127-34. [CrossRef]

2. Siegel R, Naishadham D, Jemal A. Cancer statistics, 2013. CA Cancer J Clin 2013; 63(I): II-30 [CrossRef]

3. Nomelini RS, de Carvalho Oliveira LJ, Tavares-Murta BM, Murta EFC. Parameters of blood count and tumor markers: a retrospective analysis and relation to prognostic factors in ovarian cancer. Eur $\mathrm{J}$ Gynaecol Oncol 2017; 38(3): 364-7.

4. Mironov S, Akin O, Pandit-Taskar N, Hann LE. Ovarian Cancer. Radiol Clin North Am 2007; (I): 149-66. [CrossRef]

5. Zhao Z, Xinrui Z, Jingjing L, Xue J, Peishu L, Mao H. Prognostic roles of neutrophil to lymphocyte ratio and platelet to lymphocyte ratio in ovarian cancer: a meta-analysis of retrospective studies. Arch of Gynecol Obstet 2018; 297(4): 849-57. [CrossRef]

6. Miao Y, Yan Q, Li S, Li B, Feng Y. Neutrophil to lymphocyte ratio and platelet to lymphocyte ratio are predictive of chemotherapeutic response and prognosis in epithelial ovarian cancer patients treated with platinum-based chemotherapy. Cancer Biomark 2016; 17(I): 3340. [CrossRef]

7. Seidman JD, Kurman RJ. Pathology of ovarian carcinoma. Hematol Oncol Clin North Am 2003; 17(4): 909-25. [CrossRef]

8. Skírnisdóttir l, Garmo H, Wilander E, Holmberg L. Borderline ovarian tumors in Sweden 1960-2005: trends in incidence and age at diagnosis compared to ovarian cancer. Int J Cancer 2008; 123(8): 1897901. [CrossRef]

9. Ayhan A, Celik H, Taskiran C, Bozdag G, Aksu T, et al. Oncologic and reproductive outcome after fertility-saving surgery in ovarian cancer. Eur J Gynaecol Oncol 2003; 24(3-4): 223-32.

10. Daraï E, Fauvet R, Uzan C, Gouy S, Duvillard P, Morice P, et al. Fertility and borderline ovarian tumor: a systematic review of conservative management, risk of recurrence and alternative options. Hum Reprod Update 2013; 19(2): 151-66. [CrossRef]

II. Suh-Burgmann E. Long-term outcomes following conservative surgery for borderline tumor of the ovary: a large population-based study. Gynecol Oncol 2006; 103(3): 841-7. [CrossRef]

12. Orditura M, Galizia G, Diana A, Saccone C, Cobellis L, Ventriglia J, et al. Neutrophil to lymphocyte ratio (NLR) for prediction of distant metastasis-free survival (DMFS) in early breast cancer: a propensity score-matched analysis. ESMO Open 2016; I(2): e000038. [CrossRef]

13. Liang HG, Gao K, Jia R, Li J, Wang C. Prognostic significance of the combination of preoperative fibrinogen and the neutrophillymphocyte ratio in patients with nonsmall cell lung cancer following surgical resection. Oncol LetT 2019; 17(2): 1435-44.

14. Hefler LA, Concin N, Hofstetter G, Marth C, Mustea A, Sehouli J, et al. Serum $C$-reactive protein as independent prognostic variable in patients with ovarian cancer. Clin Cancer Res 2008; 14(3): 710-4. [CrossRef]

15. Wang $Y$, Liu $P, X \cup Y$, Zhang $W$, Tong $L$, Guo Z, et al. Preoperative neutrophil-to-lymphocyte ratio predicts response to first-line platinum-based chemotherapy and prognosis in serous ovarian cancer. Cancer Chemother Pharmacol 20I5; 75(2): 255-62. [CrossRef] 
16. Zhang WW, Liu KJ, Hu GL, Liang WJ. Preoperative platelet/lymphocyte ratio is a superior prognostic factor compared to other systemic inflammatory response markers in ovarian cancer patients. Tumour Biol 2015; 36(II): 8831-7. [CrossRef]

17. Khatib G, Soysal C, Çetin C, Güleç ÜK, Güzel AB, Keskin N, et al. Does preoperative neutrophil to lymphocyte or platelet to lymphocyte ratios have a role in predicting borderline ovarian tumors? J Ovarian Res 2016; 9(I): 78. [CrossRef]

18. Williams KA, Labidi-Galy SI, Terry KL, Vitonis AF, Welch WR, Goodman $A$, et al. Prognostic significance and predictors of the neutrophil-to-lymphocyte ratio in ovarian cancer. Gynecol Oncol 2014; 132(3): 542-50. [CrossRef]

19. Asher $\vee$, Lee J, Innamaa A, Bali A. Preoperative platelet lymphocyte ratio as an independent prognostic marker in ovarian cancer. Clin Transl Oncol 20II; 13(7): 499-503. [CrossRef]

20. Topcu HO, Guzel Al, Ozer I, Kokanali MK, Gokturk U, Muftuoglu KH, et al. Comparison of neutrophil/lymphocyte and platelet/lymphocyte ratios for predicting malignant potential of suspicious ovarian masses in gynecology practice. Asian Pac J Cancer Prev 20|4; 15(I5): 6239-41. [CrossRef]

21. Yildirim M, Demir Cendek B, Filiz Avsar A. Differentiation between benign and malignant ovarian masses in the preoperative period using neutrophil-to-lymphocyte and platelet-to-lymphocyte ratios. Mol Clin Oncol 2015; 3(2): 317-2I. [CrossRef]

22. Zhu Y, Zhou S, Liu Y, Zhai L, Sun X. Prognostic value of systemic inflammatory markers in ovarian Cancer: a PRISMA-compliant meta-analysis and systematic review. BMC Cancer 2018; I8(I): 443. [CrossRef]

23. Templeton A, McNamara M, Šeruga B, Vera-Badillo F, Aneja P, Ocaña $A$, et al. Prognostic role of neutrophil-to-lymphocyte ratio in solid tumors: a systematic review and meta-analysis. J Natl Cancer Inst 2014; 106(6): djul24. [CrossRef]

24. Seckin KD, Karsli MF, Yucel B, Bestel M, Yildirim D, Canaz E, et al. The utility of tumor markers and neutrophil lymphocyte ratio in patients with an intraoperative diagnosis of mucinous borderline ovarian tumor. Eur J Obstet Gynecol Reprod Biol 2016; 196: 60-3. [CrossRef]
25. Tamakoshi K, Kikkawa F, Shibata K, Tomoda K, Obata NH, Wakahara $\mathrm{F}$, et al, Clinical value of CAI25, CA19-9, CEA, CA72-4, and TPA in borderline ovarian tumor. Gynecol Oncol 1996; 62(I): 67-72. [CrossRef]

26. Gotlieb WH, Soriano D, Achiron R, Zalel Y, Davidson B, Kopolovic $J$, et al. CA 125 measurement and ultrasonography in borderline tumors of the ovary. Am J Obstet Gynecol 2000; 183(3): 54I-6. [CrossRef]

27. Zanetta G, Rota S, Lissoni A, Meni A, Brancatelli G, Buda A. Ultrasound, physical examination, and CA 125 measurement for the detection of recurrence after conservative surgery for early borderline ovarian tumors. Gynecol Oncol 200I; 8I(I): 63-6. [CrossRef]

28. Bozkurt M, Yumru AE, Aral I. Evaluation of the importance of the serum levels of CA-125, CAI5-3, CA-19-9, carcinoembryonic antigen and alpha fetoprotein for distinguishing benign and malignant adnexal masses and contribution of different test combinations to diagnostic accuracy. Eur J Gynaecol Oncol 20I3; 34(6): 540-4.

29. Ochiai K, Shinozaki H, Takada A. A retrospective study of 1069 epithelial borderline malignancies of the ovary treated in Japan. Proceedings of Annual Meeting of the American Society of Clinical Oncology 1998; 17: Al429

30. Tempfer CB, Polteraver S, Bentz EK, Reinthaller A, Hefler LA. Accuracy of intraoperative frozen section analysis in borderline tumors of the ovary: a retrospective analysis of 96 cases and review of the literature. Gynecol Oncol 2007; 107(2): 248-52. [CrossRef]

31. Yildirim MA, Seckin KD, Togrul C, Baser E, Karsli MF, Gungor T, et al. Roles of neutrophil/lymphocyte and platelet/lymphocyte ratios in the early diagnosis of malignant ovarian masses. Asian Pac $J$ Cancer Prev 2014; 15(I6): 688I-5. [CrossRef]

32. Cho H, Hur HW, Kim SW, Kim SH, Kim JH, Kim YT, et al. Pre-treatment neutrophil to lymphocyte ratio is elevated in epithelial ovarian cancer and predicts survival after treatment. Cancer Immunol Immunother 2009; 58(I): 15-23. [CrossRef]

33. Polat M, Senol T, Ozkaya E, Ogurlu Pakay G, Cikman MS, Konukcu B, et al. Neutrophil to lymphocyte and platelet to lymphocyte ratios increase in ovarian tumors in the presence of frank stromal invasion. Clin TransI Oncol 2016; 18(5): 457-63. [CrossRef] 